\title{
A note on Caristi-type cyclic maps: related results and applications
}

\author{
Wei-Shih Du ${ }^{1 *}$ and Erdal Karapinar ${ }^{2}$
}

\author{
"Correspondence: \\ wsdu@nknucc.nknu.edu.tw \\ 'Department of Mathematics, \\ National Kaohsiung Normal \\ University, Kaohsiung, 824, Taiwan \\ Full list of author information is \\ available at the end of the article
}

\begin{abstract}
In this note, we first introduce the concept of Caristi-type cyclic map and present a new convergence theorem and a best proximity point theorem for Caristi-type cyclic maps. It should be mentioned that in our results, the dominated functions need not possess the lower semicontinuity property. Some best proximity point results and convergence theorems in the literature have been derived from our main results. Consequently, the presented results improve, extend and generalize some of the existence results on the topic.
\end{abstract}

MSC: $37 \mathrm{C} 25 ; 47 \mathrm{H} 09 ; 45 \mathrm{H} 10 ; 54 \mathrm{H} 25$

Keywords: best proximity point; Caristi-type cyclic map; $\mathcal{M} \mathcal{T}$-function ( $\mathcal{R}$-function); $\mathcal{M T}$-cyclic contraction; Caristi-type fixed point theorem

\section{Introduction and preliminaries}

Cyclic maps were introduced by Kirk, Srinavasan and Veeramani [1] in 2003 to extend the celebrated Banach contraction principle [2]: In a complete metric space $(X, d)$, every contraction $T: X \rightarrow X$ has a unique fixed point. In this principle, the mapping $T$ is necessarily continuous. In the context of cyclic mapping, the authors [1] observed the analog of Banach contraction principle for discontinuous mapping. Cyclic maps and related fixed point theorems have been investigated densely by a number of authors who have been interested in nonlinear analysis.

Let $A$ and $B$ be nonempty subsets of a nonempty set $S$. A map $T: A \cup B \rightarrow A \cup B$ is called a cyclic map if $T(A) \subseteq B$ and $T(B) \subseteq A$. Let $(X, d)$ be a metric space and $T: A \cup B \rightarrow A \cup B$ be a cyclic map. We denote the distance of the nonempty subsets $A$ and $B$ of $X$ by

$$
\operatorname{dist}(A, B)=\inf \{d(x, y): x \in A, y \in B\} .
$$

A point $x \in A \cup B$ is said to be a best proximity point for $T$ if $d(x, T x)=\operatorname{dist}(A, B)$. A map $T: A \cup B \rightarrow A \cup B$ is called a cyclic contraction if the following conditions hold:

(i) $T(A) \subseteq B$ and $T(B) \subseteq A$;

(ii) There exists $k \in(0,1)$ such that $d(T x, T y) \leq k d(x, y)+(1-k) \operatorname{dist}(A, B)$ for all $x \in A, y \in B$.

In the last decade, a number of generalizations in various directions on the existence and uniqueness of a best proximity point were investigated by several authors (see, e.g., [1, 3-13] and references therein). In particular, Eldred and Veeramani [3] obtained the following interesting best proximity point result. 
Theorem 1.1 ([3, Proposition 3.2]) Let $A$ and $B$ be nonempty closed subsets of a complete metric space $X$. Let $T: A \cup B \rightarrow A \cup B$ be a cyclic contraction map, $x_{1} \in A$ and define $x_{n+1}=T x_{n}, n \in \mathbb{N}$. Suppose that $\left\{x_{2 n-1}\right\}$ has a convergent subsequence in $A$. Then there exists $x \in A$ such that $d(x, T x)=\operatorname{dist}(A, B)$.

In 1976, Caristi [14] proved the following remarkable result that is one of the most valuable and applicable generalizations of the Banach contraction principle.

Theorem 1.2 (Caristi's fixed point theorem [14]) Let $(X, d)$ be a complete metric space and $f: X \rightarrow \mathbb{R}$ be a lower semicontinuous and bounded below function. Suppose that $T$ is a Caristi-type map on $X$ dominated by $f$; that is, $T$ satisfies

$$
d(x, T x) \leq f(x)-f(T x) \quad \text { for each } x \in X .
$$

Then $T$ has a fixed point in $X$.

Caristi's fixed point theorem has various applications in nonlinear sciences since it is an important diversity and equivalence of Ekeland's variational principle $[15,16]$ and Takahashi's nonconvex minimization theorem $[17,18]$. Due to its application potential, Caristi's fixed point theorem has been investigated, extended, generalized and improved in various ways by several authors; see, e.g., [17-26] and references therein. Very recently, in [25], the first author established some new fixed point theorems for Caristi-type maps. Indeed, the author [25] considered some suitable generalized distances without assuming that the dominated functions possess the lower semicontinuity property. More precisely, he utilized the new versions of Caristi-type fixed point theorem to deal with the existence results for any map $T$ satisfying

$$
d(T x, T y) \leq \alpha(d(x, y)) d(x, y) \quad \text { for all } x, y \in X,
$$

where $\alpha:[0, \infty) \rightarrow[0,1)$ is a function satisfying $\lim \sup _{s \rightarrow t^{+}} \alpha(s)<1$ for all $t \in[0, \infty)$; for more detail, one can refer to [25].

In this note, we first introduce the concept of Caristi-type cyclic map and present a new convergence theorem to achieve a best proximity point theorem for Caristi-type cyclic maps. It should be mentioned that in this paper we remove the lower semicontinuity property of the dominated functions in Caristi-type cyclic maps. As interesting applications of our results, we show that some results in the literature, such as $[3,12]$ and others, are concluded from both the new convergence theorem and the best proximity point theorem. The results of this paper extend, improve and generalize some well-known results on the topic in the literature.

\section{New results for Caristi-type cyclic maps}

Throughout this paper, we denote by $\mathbb{N}$ and $\mathbb{R}$ the sets of positive integers and real numbers, respectively. Let $(X, d)$ be a metric space. An extended real-valued function $\phi: X \rightarrow(-\infty,+\infty]$ is said to be lower semicontinuous (l.s.c. for short) at $w \in X$ if for any sequence $\left\{x_{n}\right\}$ in $X$ with $x_{n} \rightarrow w$ as $n \rightarrow \infty$, we have $\phi(w) \leq \liminf _{n \rightarrow \infty} \phi\left(x_{n}\right)$. The function $\phi$ is called to be l.s.c. on $X$ if $\phi$ is l.s.c. at every point of $X$. The function $\phi$ is said to be proper if $\phi \not \equiv+\infty$. 
In this paper, we first introduce the concept of Caristi-type cyclic map.

Definition 2.1 Let $A$ and $B$ be nonempty subsets of a metric space $(X, d)$ and $f: A \cup B \rightarrow$ $(-\infty,+\infty]$ and $\varphi: \mathbb{R} \rightarrow(0,+\infty)$ be functions. A self-map $T: A \cup B \rightarrow A \cup B$ is called a Caristi-type cyclic map dominated by $f$ and $\varphi$ on $A \cup B$ if the following conditions are satisfied :

(CC1) $T(A) \subseteq B$ and $T(B) \subseteq A$,

(CC2) $d(x, T x)-\operatorname{dist}(A, B) \leq \varphi(f(x))(f(x)-f(T x))$ for all $x \in A \cup B$.

In particular, if condition (CC2) is replaced with the following condition:

(CC3) $d(x, T x)-\operatorname{dist}(A, B) \leq f(x)-f(T x)$ for all $x \in A \cup B$ (that is, $\varphi(t)=1$ for all $t \in \mathbb{R}$ in $(\mathrm{CC} 2))$,

then $T$ is called a Caristi-type cyclic map dominated by $f$ on $A \cup B$.

Example 2.1 Let $X=(-10,10)$ with the usual metric $d(x, y)=|x-y|$. Then $(X, d)$ is a metric space. Let $A=[1,5]$ and $B=[-5,-1]$ be nonempty subsets of $(X, d)$. Clearly, $\operatorname{dist}(A, B)=2$. Let $f: A \cup B \rightarrow \mathbb{R}$ and $\varphi: \mathbb{R} \rightarrow(0,+\infty)$ be defined by

$$
f(x)= \begin{cases}\frac{1}{2} x-12 & \text { if } x \in[1,3) \\ 50-8 x & \text { if } x \in[3,5] \\ \frac{1}{4} x-12 & \text { if } x \in(-3,-1] \\ 48-15 x & \text { if } x \in[-5,-3]\end{cases}
$$

and

$$
\varphi(t)=10 \quad \text { for all } t \in \mathbb{R},
$$

respectively. Let $T: A \cup B \rightarrow A \cup B$ be defined by

$$
T x=-x \text { for all } x \in A \cup B \text {. }
$$

Then $T(A) \subseteq B$ and $T(B) \subseteq A$ (i.e., (CC1) holds). We claim that $T$ is a Caristi-type cyclic map dominated by $f$ and $\varphi$, but not a Caristi-type cyclic map dominated by $f$. We consider the following four possible cases.

Case 1. For $x \in[1,3)$, we have

$$
\begin{aligned}
d(x, T x)-\operatorname{dist}(A, B) & =2 x-2 \\
& <10\left(\frac{1}{4} x\right) \\
& =\varphi(f(x))(f(x)-f(T x)) .
\end{aligned}
$$

Case 2. For $x \in[3,5]$, we have

$$
\begin{aligned}
d(x, T x)-\operatorname{dist}(A, B) & =2 x-2 \\
& <10(7 x+2) \\
& =\varphi(f(x))(f(x)-f(T x)) .
\end{aligned}
$$


Case 3. For $x \in(-3,-1]$, we have

$$
\begin{aligned}
d(x, T x)-\operatorname{dist}(A, B) & =-2 x-2<10\left(-\frac{1}{4} x\right) \\
& =\varphi(f(x))(f(x)-f(T x)) .
\end{aligned}
$$

Case 4. For $x \in[-5,-3]$, we have

$$
\begin{aligned}
d(x, T x)-\operatorname{dist}(A, B) & =-2 x-2<10(-7 x-2) \\
& =\varphi(f(x))(f(x)-f(T x)) .
\end{aligned}
$$

By Cases 1-4, we verify that

$$
d(x, T x)-\operatorname{dist}(A, B) \leq \varphi(f(x))(f(x)-f(T x)) \quad \text { for all } x \in A \cup B,
$$

that is, (CC2) holds. So, $T$ is a Caristi-type cyclic map dominated by $f$ and $\varphi$. Notice that

$$
d(x, T x)-\operatorname{dist}(A, B)=2 x-2>\frac{1}{4} x=f(x)-f(T x) \quad \text { for all } x \in[2,3),
$$

which means that (CC3) does not hold. Therefore $T$ is not a Caristi-type cyclic map dominated by $f$.

The following convergence theorem is one of the main results of this paper.

Theorem 2.1 Let $A$ and $B$ be nonempty subsets of a metric space $(X, d)$. Assume that $\varphi$ : $\mathbb{R} \rightarrow(0,+\infty)$ is a nondecreasing function and $f: A \cup B \rightarrow(-\infty,+\infty]$ is a proper function which is bounded below. If $T: A \cup B \rightarrow A \cup B$ is a Caristi-type cyclic map dominated by $f$ and $\varphi$, then for any $u \in A \cup B$ with $f(u)<+\infty$, the sequence $\left\{x_{n}\right\}_{n \in \mathbb{N}}$ in $A \cup B$ defined by $x_{1}=u$ and $x_{n+1}=T x_{n}$ for $n \in \mathbb{N}$ satisfies the following conditions:

(a) $f\left(x_{n+1}\right) \leq f\left(x_{n}\right)<+\infty$ for each $n \in \mathbb{N}$,

(b) $d\left(x_{n}, x_{n+1}\right)-\operatorname{dist}(A, B) \leq \varphi\left(f\left(x_{n}\right)\right)\left(f\left(x_{n}\right)-f\left(x_{n+1}\right)\right)$ for all $n \in \mathbb{N}$,

(c) $\lim _{n \rightarrow \infty} d\left(x_{n}, x_{n+1}\right)=\operatorname{dist}(A, B)$.

Proof Let $S=\{x \in A \cup B: f(x)<+\infty\}$. Since $f$ is proper, $S \neq \emptyset$. Let $u \in S$. Define $x_{1}=u$ and $x_{n+1}=T x_{n}=T^{n} u$ for each $n \in \mathbb{N}$. Clearly, we have $f\left(x_{1}\right)<+\infty$ for $x_{1}=u$. Without loss of generality, we may assume $x_{1} \in A$. By (CC1), we have $x_{2 n-1} \in A$ and $x_{2 n} \in B$ for all $n \in \mathbb{N}$. Clearly,

$$
\operatorname{dist}(A, B) \leq d\left(x_{n}, x_{n+1}\right) \quad \text { for all } n \in \mathbb{N} .
$$

From $(\mathrm{CC} 2)$ we have

$$
\begin{aligned}
d\left(x_{1}, x_{2}\right)-\operatorname{dist}(A, B) & =d\left(x_{1}, T x_{1}\right)-\operatorname{dist}(A, B) \\
& \leq \varphi\left(f\left(x_{1}\right)\right)\left(f\left(x_{1}\right)-f\left(T x_{1}\right)\right) \\
& =\varphi\left(f\left(x_{1}\right)\right)\left(f\left(x_{1}\right)-f\left(x_{2}\right)\right),
\end{aligned}
$$


which implies

$$
f\left(x_{2}\right) \leq f\left(x_{1}\right)<+\infty \text {. }
$$

Similarly, we have

$$
d\left(x_{2}, x_{3}\right)-\operatorname{dist}(A, B) \leq \varphi\left(f\left(x_{2}\right)\right)\left(f\left(x_{2}\right)-f\left(x_{3}\right)\right)
$$

and

$$
f\left(x_{3}\right) \leq f\left(x_{2}\right) \leq f\left(x_{1}\right)<+\infty
$$

Hence, by induction, we can obtain the following inequalities:

$$
d\left(x_{n}, x_{n+1}\right)-\operatorname{dist}(A, B) \leq \varphi\left(f\left(x_{n}\right)\right)\left(f\left(x_{n}\right)-f\left(x_{n+1}\right)\right)
$$

and

$$
f\left(x_{n+1}\right) \leq f\left(x_{n}\right)<+\infty \quad \text { for each } n \in \mathbb{N} .
$$

Consequently, we have that (a) and (b) hold. Finally, let us prove (c). Since $f$ is bounded below,

$$
\gamma:=\lim _{n \rightarrow \infty} f\left(x_{n}\right)=\inf _{n \in \mathbb{N}} f\left(x_{n}\right) \text { exists. }
$$

Since $\varphi$ is nondecreasing, by (2.3), we have

$$
\varphi\left(f\left(x_{n}\right)\right) \leq \varphi\left(f\left(x_{1}\right)\right) \quad \text { for all } n \in \mathbb{N} .
$$

Taking into account (2.1), (2.2), (2.4) and (2.5), we get

$$
\operatorname{dist}(A, B) \leq d\left(x_{n}, x_{n+1}\right) \leq \varphi\left(f\left(x_{1}\right)\right)\left(f\left(x_{n}\right)-\gamma\right)+\operatorname{dist}(A, B) .
$$

Since $\lim _{n \rightarrow \infty} f\left(x_{n}\right)=\gamma$, the last inequality implies

$$
\lim _{n \rightarrow \infty} d\left(x_{n}, x_{n+1}\right)=\operatorname{dist}(A, B) .
$$

So, we conclude that (c) holds. The proof is completed.

Applying Theorem 2.1, we establish the following new best proximity point theorem for Caristi-type cyclic maps.

Theorem 2.2 Let $A$ and $B$ be nonempty subsets of a metric space $(X, d)$. Suppose that $\varphi: \mathbb{R} \rightarrow(0,+\infty)$ is a nondecreasing function and $f: A \cup B \rightarrow(-\infty,+\infty]$ is a properfunction that is bounded below. Let $u \in A$ with $f(u)<+\infty$ and $T: A \cup B \rightarrow A \cup B$ be a Caristitype cyclic map dominated by $f$ and $\varphi$. Define a sequence $\left\{x_{n}\right\}_{n \in \mathbb{N}}$ in $A \cup B$ by $x_{1}=u$ and $x_{n+1}=T x_{n}$ for $n \in \mathbb{N}$. Suppose that one of the following conditions is satisfied: 
(H1) $T$ is continuous on $A \cup B$;

$(\mathrm{H} 2) d(T x, T y) \leq d(x, y)$ for any $x \in A$ and $y \in B$;

(H3) The map $g: X \rightarrow[0, \infty)$ defined by $g(x)=d(x, T x)$ is l.s.c.

Then the following statements hold.

(a) If $\left\{x_{2 n-1}\right\}$ has a convergent subsequence in $A$, then there exists $v \in A$ such that $d(v, T v)=\operatorname{dist}(A, B)$.

(b) If $\left\{x_{2 n}\right\}$ has a convergent subsequence in $B$, then there exists $v \in B$ such that $d(v, T v)=\operatorname{dist}(A, B)$.

Proof Applying Theorem 2.1, we have

$$
\lim _{n \rightarrow \infty} d\left(x_{n}, x_{n+1}\right)=\operatorname{dist}(A, B) .
$$

Since $x_{1}=u \in A$, we have $x_{2 n-1} \in A$ and $x_{2 n} \in B$ for all $n \in \mathbb{N}$. Let us prove the conclusion (a). Assume that $\left\{x_{2 n-1}\right\}$ has a convergent subsequence $\left\{x_{2 n_{k}-1}\right\}$ in $A$. Hence there exists $v \in A$ such that $x_{2 n_{k}-1} \rightarrow v$ as $k \rightarrow \infty$ or

$$
\lim _{k \rightarrow \infty} d\left(v, x_{2 n_{k}-1}\right)=0 .
$$

Clearly, we have

$$
\operatorname{dist}(A, B) \leq d\left(v, x_{2 n_{k}}\right) \leq d\left(v, x_{2 n_{k}-1}\right)+d\left(x_{2 n_{k}-1}, x_{2 n_{k}}\right) \quad \text { for all } k \in \mathbb{N} \text {. }
$$

Taking into account (2.6), (2.7) and (2.8), we get

$$
\lim _{n \rightarrow \infty} d\left(v, x_{2 n_{k}}\right)=\operatorname{dist}(A, B) .
$$

Now, we verify $d(v, T v)=\operatorname{dist}(A, B)$. Suppose that (H1) holds. By the continuity of $T$, we derive

$$
x_{2 n_{k}}=T x_{2 n_{k}-1} \rightarrow T \nu \quad \text { as } k \rightarrow \infty .
$$

By (2.9) and (2.10), we get $d(v, T v)=\operatorname{dist}(A, B)$.

If (H2) holds, since

$$
\operatorname{dist}(A, B) \leq d\left(T v, x_{2 n_{k}+1}\right) \leq d\left(v, x_{2 n_{k}}\right) \quad \text { for all } k \in \mathbb{N}
$$

and (2.9), we find that $d(v, T v)=\operatorname{dist}(A, B)$.

Finally, assume that (H3) holds. By the lower semicontinuity of $g, x_{2 n_{k}-1} \rightarrow v$ as $k \rightarrow \infty$ and (2.6), we obtain

$$
\begin{aligned}
\operatorname{dist}(A, B) & \leq d(v, T v) \\
& =g(v) \\
& \leq \liminf _{k \rightarrow \infty} g\left(x_{2 n_{k}-1}\right) \\
& =\lim _{k \rightarrow \infty} d\left(x_{2 n_{k}-1}, x_{2 n_{k}}\right)=\operatorname{dist}(A, B),
\end{aligned}
$$

which implies $d(v, T v)=\operatorname{dist}(A, B)$. 
Following a similar argument as in the proof of (a), one can also show the desired conclusion (b).

Here, we give an example illustrating Theorem 2.2.

Example 2.2 Let $X, A, B, f, \varphi$ and $T$ be the same as in Example 2.1. Hence $T$ is a Caristitype cyclic map dominated by $f$ and $\varphi$. Note that $f$ is not lower semicontinuous at $x=3$ and -3 , so $f$ is not lower semicontinuous on $A \cup B$. Since $f(x) \geq-100$ for all $x \in A \cup B, f$ is a bounded below function on $A \cup B$. By the definition of $T$, we know that $T$ is continuous on $A \cup B$. Hence (H1) as in Theorem 2.2 holds. It is obvious that

$$
d(1, T(1))=d(-1, T(-1))=2=\operatorname{dist}(A, B) .
$$

On the other hand, let $x_{1}=1 \in A$ and $x_{n+1}=T x_{n}$ for $n \in \mathbb{N}$. Then we have $x_{2 n-1}=1 \in A$ and $x_{2 n}=-1 \in B$ for all $n \in \mathbb{N}$. So $\left\{x_{2 n-1}\right\}$ and $\left\{x_{2 n}\right\}$ have convergent subsequences in $A$ and $B$, respectively. Therefore, all the assumptions of Theorem 2.2 are satisfied. Applying Theorem 2.2, we also prove that there exist $v \in A$ and $w \in B$ (precisely speaking, $v=1$ and $w=-1)$ such that $d(v, T v)=d(w, T w)=\operatorname{dist}(A, B)$.

\section{Some applications}

A function $\alpha:[0, \infty) \rightarrow[0,1)$ is said to be an $\mathcal{M T}$-function (or $\mathcal{R}$-function) [12, 25-30] if $\lim \sup _{s \rightarrow t^{+}} \alpha(s)<1$ for all $t \in[0, \infty)$. It is obvious that if $\alpha:[0, \infty) \rightarrow[0,1)$ is a nondecreasing function or a nonincreasing function, then $\alpha$ is an $\mathcal{M T}$-function. So the set of $\mathcal{M T}$-functions is a rich class.

Recently, $\mathrm{Du}$ [28] first proved the following characterizations of $\mathcal{M T}$-functions which are quite useful for proving our main results.

Theorem $\mathbf{D}([28$, Theorem 2.1]) Let $\alpha:[0, \infty) \rightarrow[0,1)$ be a function. Then the following statements are equivalent.

(a) $\alpha$ is an $\mathcal{M T}$-function.

(b) For each $t \in[0, \infty)$, there exist $r_{t}^{(1)} \in[0,1)$ and $\varepsilon_{t}^{(1)}>0$ such that $\alpha(s) \leq r_{t}^{(1)}$ for all $s \in\left(t, t+\varepsilon_{t}^{(1)}\right)$.

(c) For each $t \in[0, \infty)$, there exist $r_{t}^{(2)} \in[0,1)$ and $\varepsilon_{t}^{(2)}>0$ such that $\alpha(s) \leq r_{t}^{(2)}$ for all $s \in\left[t, t+\varepsilon_{t}^{(2)}\right]$.

(d) For each $t \in[0, \infty)$, there exist $r_{t}^{(3)} \in[0,1)$ and $\varepsilon_{t}^{(3)}>0$ such that $\alpha(s) \leq r_{t}^{(3)}$ for all $s \in\left(t, t+\varepsilon_{t}^{(3)}\right]$.

(e) For each $t \in[0, \infty)$, there exist $r_{t}^{(4)} \in[0,1)$ and $\varepsilon_{t}^{(4)}>0$ such that $\alpha(s) \leq r_{t}^{(4)}$ for all $s \in\left[t, t+\varepsilon_{t}^{(4)}\right)$.

(f) For any nonincreasing sequence $\left\{x_{n}\right\}_{n \in \mathbb{N}}$ in $[0, \infty)$, we have $0 \leq \sup _{n \in \mathbb{N}} \alpha\left(x_{n}\right)<1$.

(g) $\alpha$ is a function of contractive factor; that is, for any strictly decreasing sequence $\left\{x_{n}\right\}_{n \in \mathbb{N}}$ in $[0, \infty)$, we have $0 \leq \sup _{n \in \mathbb{N}} \alpha\left(x_{n}\right)<1$.

Let us recall the concept of $\mathcal{M T}$-cyclic contractions introduced first by Du and Lakzian [12].

Definition 3.1 [12] Let $A$ and $B$ be nonempty subsets of a metric space $(X, d)$. If a map $T: A \cup B \rightarrow A \cup B$ satisfies 
(MT1) $T(A) \subseteq B$ and $T(B) \subseteq A$;

(MT2) there exists an $\mathcal{M T}$-function $\alpha:[0, \infty) \rightarrow[0,1)$ such that

$$
d(T x, T y) \leq \alpha(d(x, y)) d(x, y)+(1-\alpha(d(x, y))) \operatorname{dist}(A, B) \quad \text { for any } x \in A \text { and } y \in B,
$$

then $T$ is called an $\mathcal{M T}$-cyclic contraction with respect to $\alpha$ on $A \cup B$.

The following example shows that there exists an $\mathcal{M T}$-cyclic contraction which is not a cyclic contraction.

Example 3.1 [12] Let $X=\left\{v_{1}, v_{2}, v_{3}, \ldots\right\}$ be a countable set and $\left\{\tau_{n}\right\}$ be a strictly increasing convergent sequence of positive real numbers. Denote by $\tau_{\infty}:=\lim _{n \rightarrow \infty} \tau_{n}$. Then $\tau_{2}<\tau_{\infty}$. Let $d: X \times X \rightarrow[0, \infty)$ be defined by $d\left(v_{n}, v_{n}\right)=0$ for all $n \in \mathbb{N}$ and $d\left(v_{n}, v_{m}\right)=d\left(v_{m}, v_{n}\right)=$ $\tau_{m}$ if $m>n$. Then $d$ is a metric on $X$. Set $A=\left\{v_{1}, v_{3}, v_{5}, \ldots\right\}, B=\left\{v_{2}, v_{4}, v_{6}, \ldots\right\}$. Define a map $T: A \cup B \rightarrow A \cup B$ by

$$
T v_{n} \stackrel{\text { def }}{=} \begin{cases}v_{2} & \text { if } n=1 \\ v_{n-1} & \text { if } n>1\end{cases}
$$

for $n \in \mathbb{N}$ and define $\alpha:[0, \infty) \rightarrow[0,1)$ by

$$
\alpha(t) \stackrel{\text { def }}{=} \begin{cases}\frac{\tau_{n-1}}{\tau_{n}} & \text { if } t=\tau_{n} \text { for some } n \in \mathbb{N} \text { with } n>2 \\ 0 & \text { otherwise. }\end{cases}
$$

Then $T$ is an $\mathcal{M T}$-cyclic contraction with respect to $\alpha$, but not a cyclic contraction on $A \cup B$.

The following result tells us the relation between an $\mathcal{M T}$-cyclic contraction and a Caristi-type cyclic map.

Theorem 3.1 Let $A$ and $B$ be nonempty subsets of a metric space $(X, d)$ and $T: A \cup B \rightarrow$ $A \cup B$ be an $\mathcal{M T}$-cyclic contraction with respect to $\alpha$. Then there exist a bounded below function $f: A \cup B \rightarrow \mathbb{R}$ and a nondecreasing function $\varphi: \mathbb{R} \rightarrow(0,+\infty)$ such that $T$ is a Caristi-type cyclic map dominated by $f$ and $\varphi$.

Proof Denote $T^{0}=I$ (the identity mapping). Let $x \in A \cup B$ be given. Define a sequence $\left\{w_{n}\right\}_{n \in \mathbb{N}}$ in $A \cup B$ by $w_{1}=x$ and $w_{n}=T w_{n}=T^{n-1} x$ for $n \in \mathbb{N}$. Clearly, the condition (MT2) implies that $T$ satisfies

$$
d(T x, T y) \leq d(x, y) \quad \text { for any } x \in A \text { and } y \in B
$$

So from the last inequality we deduce

$$
d\left(w_{n+1}, w_{n+2}\right) \leq d\left(w_{n}, w_{n+1}\right) \quad \text { for all } n \in \mathbb{N} \text {. }
$$

Hence the sequence $\left\{d\left(w_{n}, w_{n+1}\right)\right\}$ is nonincreasing in $[0, \infty)$. Since $\alpha$ is an $\mathcal{M T}$-function, by (g) of Theorem $\mathrm{D}$, we obtain

$$
0 \leq \sup _{n \in \mathbb{N}} \alpha\left(d\left(w_{n}, w_{n+1}\right)\right)<1
$$


Since $x \in A \cup B$ is arbitrary, we can define a new function $\beta: A \cup B \rightarrow[0,1)$ by

$$
\beta(x):=\sup _{n \in \mathbb{N}} \alpha\left(d\left(w_{n}, w_{n+1}\right)\right)=\sup _{n \in \mathbb{N}} \alpha\left(d\left(T^{n-1} x, T^{n} x\right)\right) \quad \text { for } x \in A \cup B .
$$

Clearly, for each $x \in A \cup B$, we have

$$
\beta(T x) \leq \beta(x)
$$

and

$$
\alpha\left(d\left(T^{n-1} x, T^{n} x\right)\right) \leq \beta(x) \quad \text { for all } n \in \mathbb{N} .
$$

Let $x \in A \cup B$ be given. Without loss of generality, we may assume $x \in A$. Then $T x \in B$. By (MT2), we get

$$
d\left(T x, T^{2} x\right)-\operatorname{dist}(A, B) \leq \alpha(d(x, T x))(d(x, T x)-\operatorname{dist}(A, B))
$$

and hence

$$
d(x, T x)-\alpha(d(x, T x))(d(x, T x)-\operatorname{dist}(A, B)) \leq d(x, T x)-d\left(T x, T^{2} x\right)+\operatorname{dist}(A, B) .
$$

By exploiting inequalities (3.1), (3.2) and (3.3), we obtain

$$
\begin{aligned}
d(x, T x)-\operatorname{dist}(A, B) & \leq \frac{1}{1-\alpha(d(x, T x))} d(x, T x)-\frac{1}{1-\alpha(d(x, T x))} d\left(T x, T^{2} x\right) \\
& \leq \frac{1}{1-\beta(x)} d(x, T x)-\frac{1}{1-\beta(T x)} d\left(T x, T^{2} x\right) .
\end{aligned}
$$

Let $\varphi: \mathbb{R} \rightarrow[0,+\infty)$ and $f: A \cup B \rightarrow \mathbb{R}$ be defined by

$$
\varphi(t)=1 \quad \text { for } t \in \mathbb{R}
$$

and

$$
f(x)=\frac{1}{1-\beta(x)} d(x, T x) \quad \text { for } x \in A \cup B,
$$

respectively. Then $\varphi$ is a nondecreasing function and $f$ is a bounded below function. Clearly, $f(x)<+\infty$ for all $x \in A \cup B$. From (3.3), we obtain

$$
d(x, T x)-\operatorname{dist}(A, B) \leq \varphi(f(x))(f(x)-f(T x)) \text { for all } x \in A \cup B,
$$

which means that $T$ is a Caristi-type cyclic map dominated by $f$ and $\varphi$.

Theorem 3.2 [12] Let $A$ and $B$ be nonempty subsets of a metric space $(X, d)$ and $T: A \cup$ $B \rightarrow A \cup B$ be an $\mathcal{M T}$-cyclic contraction with respect to $\alpha$. Then there exists a sequence $\left\{x_{n}\right\}_{n \in \mathbb{N}}$ such that

$$
\lim _{n \rightarrow \infty} d\left(x_{n}, T x_{n}\right)=\inf _{n \in \mathbb{N}} d\left(x_{n}, T x_{n}\right)=\operatorname{dist}(A, B) .
$$


Proof Applying Theorem 3.1, there exist a bounded below function $f: A \cup B \rightarrow \mathbb{R}$ and a nondecreasing function $\varphi: \mathbb{R} \rightarrow(0,+\infty)$ such that $T$ is a Caristi-type cyclic map dominated by $f$ and $\varphi$. Let $u \in A \cup B$ be given. Let $\left\{x_{n}\right\}_{n \in \mathbb{N}} \subset A \cup B$ be defined by $x_{1}=u$ and $x_{n+1}=T x_{n}$ for $n \in \mathbb{N}$. Applying Theorem 2.1, we have

$$
\lim _{n \rightarrow \infty} d\left(x_{n}, x_{n+1}\right)=\operatorname{dist}(A, B)
$$

Since the condition (MT2) implies that $T$ satisfies

$$
d(T x, T y) \leq d(x, y) \quad \text { for any } x \in A \text { and } y \in B
$$

we know that the sequence $\left\{d\left(x_{n}, x_{n+1}\right)\right\}$ is nonincreasing in $[0, \infty)$. By $(3.4)$, we get

$$
\operatorname{dist}(A, B)=\lim _{n \rightarrow \infty} d\left(x_{n}, x_{n+1}\right)=\inf _{n \in \mathbb{N}} d\left(x_{n}, x_{n+1}\right) \geq 0 .
$$

The proof is completed.

Theorem 3.3 [12] Let $A$ and $B$ be nonempty subsets of a metric space $(X, d)$ and $T: A \cup$ $B \rightarrow A \cup B$ be an $\mathcal{M T}$-cyclic contraction with respect to $\alpha$. For a given $x_{1} \in A$, define an iterative sequence $\left\{x_{n}\right\}_{n \in \mathbb{N}}$ by $x_{n+1}=T x_{n}$ for $n \in \mathbb{N}$. Suppose that $\left\{x_{2 n-1}\right\}$ has a convergent subsequence in $A$, then there exists $v \in A$ such that $d(v, T v)=\operatorname{dist}(A, B)$.

Proof Applying Theorem 3.1, there exist a bounded below function $f: A \cup B \rightarrow \mathbb{R}$ and a nondecreasing function $\varphi: \mathbb{R} \rightarrow(0,+\infty)$ such that $T$ is a Caristi-type cyclic map dominated by $f$ and $\varphi$. Let $u=x_{1} \in A$ be given. Let $\left\{x_{n}\right\}_{n \in \mathbb{N}} \subset A \cup B$ be defined by $x_{n+1}=T x_{n}$ for $n \in \mathbb{N}$. Since the condition (MT2) implies the condition (H2) as in Theorem 2.2, all the assumptions of Theorem 2.2 are satisfied. By applying (a) of Theorem 2.2, there exists $v \in A$ such that $d(v, T v)=\operatorname{dist}(A, B)$.

Remark 3.1 ([3, Proposition 3.2]) (i.e., Theorem 1.1) is a special case of Theorem 3.3.

Finally, applying Theorem 2.1, we can establish a new Caristi-type fixed point theorem without assuming that the dominated functions possess the lower semicontinuity property.

Theorem 3.4 Let $M$ be a nonempty subset of a metric space $(X, d), f: M \rightarrow(-\infty,+\infty]$ be a proper and bounded below function, $\varphi: \mathbb{R} \rightarrow(0,+\infty)$ be a nondecreasing function and $T: M \rightarrow M$ be a selfmap on $X$. Suppose that $T$ is of Caristi type on $M$ dominated by $\varphi$ and $f$, that is,

$$
d(x, T x) \leq \varphi(f(x))(f(x)-f(T x)) \quad \text { for each } x \in M .
$$

Then there exists a sequence $\left\{x_{n}\right\}_{n \in \mathbb{N}}$ in $M$ such that $\left\{x_{n}\right\}_{n \in \mathbb{N}}$ is Cauchy.

Moreover, if $(X, d)$ is complete and $M$ is closed in $X$, and one of the following conditions is satisfied:

(D1) $T$ is continuous on $M$; 
(D2) $T$ is closed, that is, $\operatorname{Gr} T=\{(x, y) \in M \times M: y=T x\}$, the graph of $T$, is closed in $M \times M$

(D3) $T$ he map $g: X \rightarrow[0, \infty)$ defined by $g(x)=d(x, T x)$ is l.s.c.

Then the mapping $T$ admits a fixed point in $X$, and for any $u \in X$ with $f(u)<+\infty$, the sequence $\left\{T^{n} u\right\}_{n \in \mathbb{N}}$ converges to a fixed point of $T$.

Proof Let $A=B=M$. Then we have $A \cup B=M, T(A) \subseteq B, T(B) \subseteq A$ and $\operatorname{dist}(A, B)=0$. So (3.5) implies

$$
d(x, T x)-\operatorname{dist}(A, B) \leq \varphi(f(x))(f(x)-f(T x)) \quad \text { for all } x \in A \cup B=M .
$$

Hence $T$ a Caristi-type cyclic map dominated by $f$ and $\varphi$ on $A \cup B$. Since $f$ is proper, there exists $u \in M=A \cup B$ such that $f(u)<+\infty$. Let $\left\{x_{n}\right\}_{n \in \mathbb{N}} \subset M$ be defined by $x_{1}=u$ and $x_{n+1}=T x_{n}$ for $n \in \mathbb{N}$. By applying Theorem 2.1, we have

(a) $f\left(x_{n+1}\right) \leq f\left(x_{n}\right)<+\infty$ for each $n \in \mathbb{N}$,

(b) $d\left(x_{n}, x_{n+1}\right) \leq \varphi\left(f\left(x_{n}\right)\right)\left(f\left(x_{n}\right)-f\left(x_{n+1}\right)\right)$ for all $n \in \mathbb{N}$,

(c) $\lim _{n \rightarrow \infty} d\left(x_{n}, x_{n+1}\right)=0$.

Since $\varphi$ is nondecreasing, by (a), we have

$$
\varphi\left(f\left(x_{n}\right)\right) \leq \varphi\left(f\left(x_{1}\right)\right) \quad \text { for all } n \in \mathbb{N} .
$$

Since $f$ is bounded below and the sequence $\left\{f\left(x_{n}\right)\right\}_{n \in \mathbb{N}}$ is nonincreasing in $[0,+\infty)$,

$$
\gamma:=\lim _{n \rightarrow \infty} f\left(x_{n}\right)=\inf _{n \in \mathbb{N}} f\left(x_{n}\right) \text { exists. }
$$

For $m>n$ with $m, n \in \mathbb{N}$, taking into account (3.5), (3.6) and (3.7), we get

$$
d\left(x_{n}, x_{m}\right) \leq \sum_{j=n}^{m-1} d\left(x_{j}, x_{j+1}\right) \leq \varphi\left(f\left(x_{1}\right)\right)\left(f\left(x_{n}\right)-\gamma\right) .
$$

Let $\lambda_{n}=\varphi\left(f\left(x_{1}\right)\right)\left(f\left(x_{n}\right)-\gamma\right), n \in \mathbb{N}$. Then

$$
\sup \left\{d\left(x_{n}, x_{m}\right): m>n\right\} \leq \lambda_{n} \quad \text { for each } n \in \mathbb{N} \text {. }
$$

Since $\lim _{n \rightarrow \infty} f\left(x_{n}\right)=\gamma, \lim _{n \rightarrow \infty} \lambda_{n}=0$. From (3.8) we obtain

$$
\lim _{n \rightarrow \infty} \sup \left\{d\left(x_{n}, x_{m}\right): m>n\right\}=0,
$$

which proves that $\left\{x_{n}\right\}_{n \in \mathbb{N}}$ is a Cauchy sequence in $M$.

Moreover, assume that $(X, d)$ is complete and $M$ is closed in $X$. So $(M, d)$ is a complete metric space. By the completeness of $M$, there exists $v_{u} \in M$ such that $x_{n} \rightarrow v_{u}$ as $n \rightarrow \infty$. We claim $v_{u} \in \mathcal{F}(T)$. If (D1) holds, since $T$ is continuous on $M, x_{n+1}=T x_{n}$ for each $n \in \mathbb{N}$ and $x_{n} \rightarrow v_{u}$ as $n \rightarrow \infty$, we get

$$
v_{u}=\lim _{n \rightarrow \infty} x_{n}=\lim _{n \rightarrow \infty} x_{n+1}=\lim _{n \rightarrow \infty} T x_{n}=T\left(\lim _{n \rightarrow \infty} x_{n}\right)=T v_{u}
$$


If (D2) holds, since $T$ is closed, $x_{n+1}=T x_{n}$ for each $n \in \mathbb{N}$ and $x_{n} \rightarrow v_{u}$ as $n \rightarrow \infty$, we have $T v_{u}=v_{u}$. Finally, assume that (D3) holds. Since $\lim _{n \rightarrow \infty} d\left(x_{n}, x_{n+1}\right)=0$, we obtain

$$
\begin{aligned}
d\left(v_{u}, T v_{u}\right) & =g\left(v_{u}\right) \\
& \leq \liminf _{n \rightarrow \infty} g\left(x_{n}\right) \\
& =\lim _{n \rightarrow \infty} d\left(x_{n}, x_{n+1}\right)=0,
\end{aligned}
$$

we obtain $d\left(v_{u}, T v_{u}\right)=0$ and hence $T v_{u}=v_{u}$. This completes the proof.

\section{Competing interests}

The authors declare that they have no competing interests.

\section{Authors' contributions}

Both authors contributed equally and significantly in writing this paper. Both authors read and approved the final manuscript.

\section{Author details}

'Department of Mathematics, National Kaohsiung Normal University, Kaohsiung, 824, Taiwan. ²Department of Mathematics, Atilim University, Incek, Ankara, Turkey.

\section{Acknowledgements}

The first author was supported by Grant No. NSC 102-2115-M-017-001 of the National Science Council of the Republic of China.

Received: 12 September 2013 Accepted: 14 November 2013 Published: 16 Dec 2013

\section{References}

1. Kirk, WA, Srinivasan, PS, Veeramani, P: Fixed points for mappings satisfying cyclical contractive conditions. Fixed Point Theory 4, 79-89 (2003)

2. Banach, S: Sur les operations dans les ensembles abstraits et leur application aux equations itegrales. Fundam. Math. 3, 133-181 (1922)

3. Anthony Eldred, A, Veeramani, P: Existence and convergence of best proximity points. J. Math. Anal. Appl. 323, 1001-1006 (2006)

4. Anthony Eldred, A, Anuradha, J, Veeramani, P: On the equivalence of the Mizoguchi-Takahashi fixed point theorem to Nadler's theorem. Appl. Math. Lett. 22, 1539-1542 (2009)

5. Al-Thagafi, MA, Shahzad, N: Convergence and existence results for best proximity points. Nonlinear Anal. 70, 3665-3671 (2009)

6. Suzuki, T, Kikkawa, M, Vetro, C: The existence of the best proximity points in metric spaces with the property UC. Nonlinear Anal. 71, 2918-2926 (2009)

7. Jleli, M, Karapinar, E, Samet, B: On best proximity points under the P-property on partially ordered metric spaces. Abstr. Appl. Anal. 2013, Article ID 150970 (2013). doi:10.1155/2013/150970

8. Karapinar, E: On best proximity point of $\psi$-Geraghty contractions. Fixed Point Theory Appl. 2013, Article ID 200 (2013). doi:10.1186/1687-1812-2013-200

9. Jleli, M, Karapinar, E, Samet, B: Best proximity points for generalized $\alpha$ - $\psi$-proximal contractive type mappings. J. Appl. Math. 2013, Article ID 534127 (2013). doi:10.1155/2013/534127

10. Karapinar, E, Petruşel, G, Tas, K: Best proximity point theorems for KT-types cyclic orbital contraction mappings. Fixed Point Theory 13(2), 537-546 (2012)

11. Karapinar, E: Best proximity points of cyclic mappings. Appl. Math. Lett. 25, 1761-1766 (2012)

12. Du, W-S, Lakzian, H: Nonlinear conditions for the existence of best proximity points. J. Inequal. Appl. 2012, Article ID 206 (2012). doi:10.1186/1029-242X-2012-206

13. Gabeleh, M, Shahzad, N: Existence and convergence theorems of best proximity points. J. Appl. Math. 2013, Article ID 101439 (2013). doi:10.1155/2013/101439

14. Caristi, J: Fixed point theorems for mappings satisfying inwardness conditions. Trans. Am. Math. Soc. 215, 241-251 (1976)

15. Ekeland, I: On the variational principle. J. Math. Anal. Appl. 47, 324-353 (1974)

16. Ekeland, I: Nonconvex minimization problems. Bull. Am. Math. Soc. 1, 443-474 (1979)

17. Takahashi, W: Existence theorems generalizing fixed point theorems for multivalued mappings. In: Théra, MA, Baillon, JB (eds.) Fixed Point Theory and Applications. Pitmam Research Notes in Mathematics Series, vol. 252, pp. 397-406. Longman, Harlow (1991)

18. Takahashi, W: Nonlinear Functional Analysis. Yokohama Publishers, Yokohama (2000)

19. Khamsi, MA: Remarks on Caristi's fixed point theorem. Nonlinear Anal. 71, 227-231 (2009)

20. Jachymski, JR: Caristi's fixed point theorem and selections of set-valued contractions. J. Math. Anal. Appl. 227, 55-67 (1998) 
21. Kirk, WA, Saliga, LM: The Brézis-Browder order principle and extensions of Caristi's theorem. Nonlinear Anal. 47, 2765-2778 (2001)

22. Petrusşel, A, Sîntămărian, A: Single-valued and multi-valued Caristi type operators. Publ. Math. (Debr.) 60, 167-177 (2002)

23. Feng, Y, Liu, S: Fixed point theorems for multi-valued contractive mappings and multi-valued Caristi type mappings. J. Math. Anal. Appl. 317, 103-112 (2006)

24. Karapinar, E: Generalizations of Caristi Kirk's theorem on partial metric spaces. Fixed Point Theory Appl. 2011, Article ID 4 (2011)

25. Du, W-S: On Caristi type maps and generalized distances with applications. Abstr. Appl. Anal. 2013, Article ID 407219 (2013). doi:10.1155/2013/407219

26. Du, W-S: On generalized weakly directional contractions and approximate fixed point property with applications. Fixed Point Theory Appl. 2012, Article ID 6 (2012). doi:10.1186/1687-1812-2012-6

27. Du, W-S: Some new results and generalizations in metric fixed point theory. Nonlinear Anal. 73, 1439-1446 (2010)

28. Du, W-S: On coincidence point and fixed point theorems for nonlinear multivalued maps. Topol. Appl. 159, 49-56 (2012)

29. Du, W-S: New existence results and generalizations for coincidence points and fixed points without global completeness. Abstr. Appl. Anal. 2013, Article ID 214230 (2013). doi:10.1155/2013/214230

30. Lin, I-J, Chen, T-H: New existence theorems of coincidence points approach to generalizations of Mizoguchi-Takahashi's fixed point theorem. Fixed Point Theory Appl. 2012, Article ID 124156 (2012). doi:10.1186/1687-1812-2012-156

10.1186/1687-1812-2013-344

Cite this article as: Du and Karapinar: A note on Caristi-type cyclic maps: related results and applications. Fixed Point Theory and Applications 2013, 2013:344

\section{Submit your manuscript to a SpringerOpen ${ }^{\circ}$ journal and benefit from:}

- Convenient online submission

- Rigorous peer review

- Immediate publication on acceptance

Open access: articles freely available online

- High visibility within the field

- Retaining the copyright to your article 